\title{
Ultra-high-precision Industrial Robots Calibration
}

\author{
Emanuele Lubrano, Dr. Mohamed Bouri and Prof. Reymond Clavel, LSRO2 - EPFL - Switzerland
}

\begin{abstract}
This paper furnishes the theoretical basis to perform the calibration of one or more ultra-high-precision industrial robots operating in the same workspace. We propose a new calibration procedure that keeps in account the factors that lower the robot accuracy at nanometer scale. To validate this approach, we present the practical case of the calibration of a two industrial robots system. Finally, we propose nanoindentation as an alternative method to evaluate the final accuracy reached by the system after calibration.
\end{abstract}

\section{INTRODUCTION}

I NDUSTRIAL robots are frequently used cooperatively along an assembly line. The current trend in research is to adapt such concept to the case of micro-production [1]. This is done by building modular micro-factories equipped with high-precision or ultra-high-precision robots. Such robots will perform industrial processes and displace the manufactured pieces in different parts of the system.

For applications where those robots cooperate at submicrometric level of accuracy, it is necessary to have a reliable calibration procedure that allows their use together.

While for standard robots it is easy to fix a common coordinate system in the workspace and to reference all the robots to it (closed-loop calibration method [2]), in the case of ultra-high-precision robots it is not possible to follow this procedure. In fact, in some cases the zero reference could not be reachable from all the robots involved in the operation. Thus, the reference of the system will drift and move in time, dependently to thermal effects acting on it.

In this article we will provide a new calibration procedure specific for ultra-high-precision robots and show the most common sources of inaccuracy that act on them. Thus, we will propose a real case study consisting in the calibration a two robots system equipped to perform nano-indentation. Finally, nano-indentation will be used to introduce the issue of the reference incertitude - in the case of one robot and its tool-tip and in the case of a two robots system - and to evaluate the final accuracy reached by the overall system.

\section{A. The Case Study}

We considered the system of two robots "Agietron Micro-

Manuscript received September 15, 2010. This work was supported in part by the Swiss National Foundation for Research (FNS) and from EPFL.

Emanuele Lubrano is member of IEEE since 2010. He is with the LSRO (Laboratory of Robotic Systems), EPFL, Lausanne, Switzerland (phone: +41 2169378 16; e-mail: emanuele.lubrano@epfl.ch).

Mohamed Bouri is senior member of IEEE since 2009. He is with the LSRO (Laboratory of Robotic Systems), EPFL, Lausanne, Switzerland (phone: +41 2169338 16; e-mail: mohamed.bouri@epfl.ch).

Professor Reymond Clavel is the director of the LSRO, EPFL, Lausanne, Switzerland (e-mail: reymond.clavel@epfl.ch).
Nano" and "MinAngle" (fig.1), a system designed to perform the $\mu$-EDM process (Electro Discharge Machining). The complete system fills up a volume of $\sim 32 \times 32 \times 50 \mathrm{~cm}$.

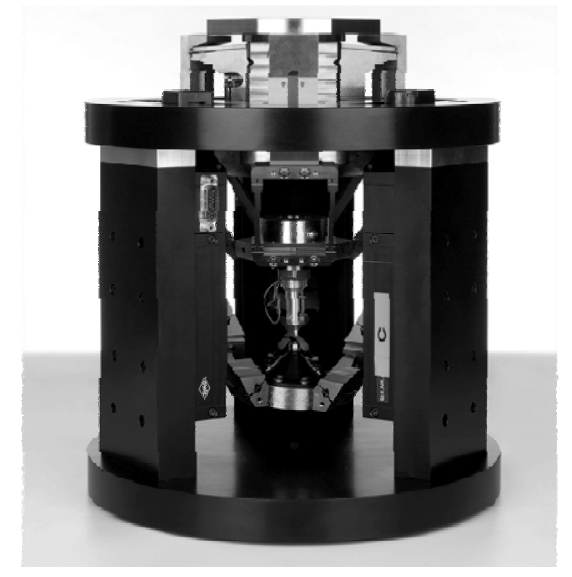

Fig. 1. A picture of the two robots system composed by the Agietron Micro-Nano (on top) and the MinAngle (down).

The Agietron Micro-Nano has a delta parallel kinematic [3] (fig. 2), flexure hinges joints, encoders with a resolution of $10 \mathrm{~nm}, 3$ translational DOF (degree(s)-of-freedom) and it has been built in titanium. Thanks to those features, the robot is fast, stiff and rigid. It is currently used in industry to perform the $\mu$-EDM process [4]. The robot operates in a working volume of $\sim 1 \mathrm{~cm}^{3}$.

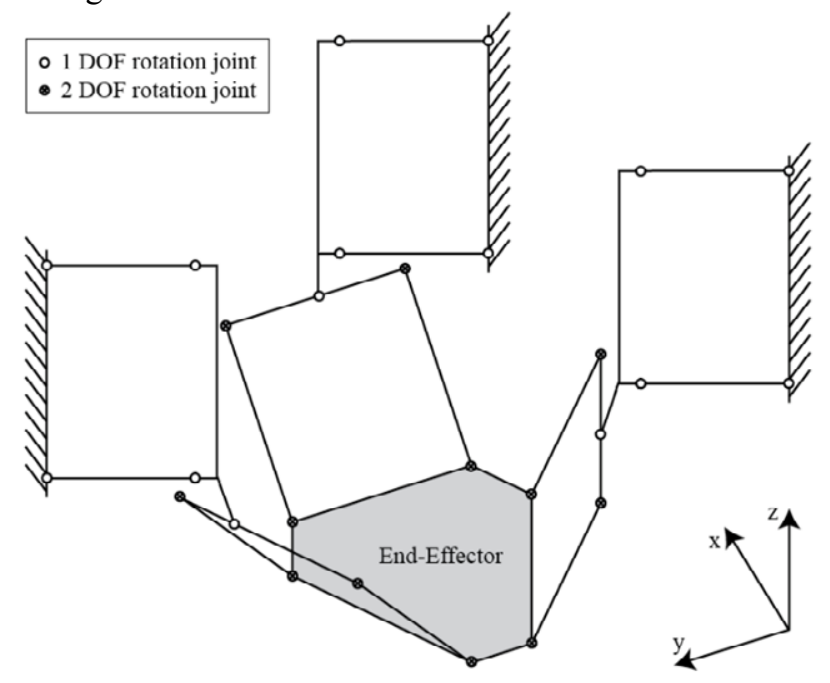

Fig. 2. Kinematic chain of the robot Agietron Micro-Nano

The MinAngle has been built to be the left-hand of the Agietron Micro-Nano [5]. It has been developed to hold the piece manufactured by the Agietron Micro-Nano and rotate 
it around the two horizontal axes. It is a parallel kinematic 3 DOF robot (1 translation and 2 rotations) equipped with flexure hinges joints and built in titanium (fig. 3). It has a course in rotation of $\pm 15^{\circ}$ and a course in translation of \pm 5 $\mathrm{mm}$. It has a linear resolution of $0.25 \mu \mathrm{m}$ and an angular resolution of 0.5 arcsec.

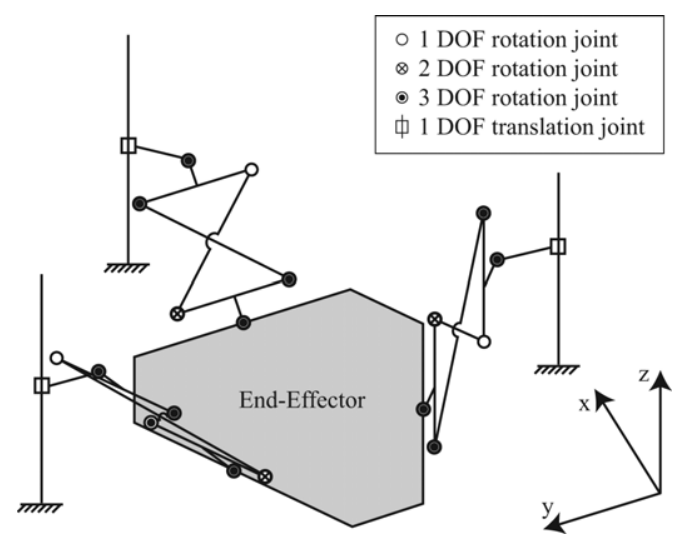

Fig. 3. Kinematic chain of the robot MinAngle.

The two robot together have a total of 5 DOF (the vertical axis is redundant). Moreover, the Agietron Micro-Nano can compensate the parasitic translations of the MinAngle, while the MinAngle can correct the parasitic rotations of the Agietron Micro-Nano.

\section{B. The Processes Involving the System}

$\mu-E D M$ is a manufacturing process used for cutting complex shapes and thin walled configurations without distortion. It is recommended for hard materials or for materials typically machined by grinding [6]. It is suited for applications characterized by extremely exacting tolerances (accuracy $\sim 1 \mu \mathrm{m}$ ). Since it is a contactless process, it is also well suited for making fragile parts that cannot take the stress of a normal machining process. To perform it, an electrode or a wire is mounted on the robot end-effector. A controlled electrical spark is used to erode away from the manufactured object any material that can conduct electricity. A series of discharges takes places between the electrode and the conductor while the robot is moving along the desired trajectory.

Indentation is a process normally used for determining the mechanical properties of materials [7]. Since it is more accurate $\mu$-EDM, it has been chosen to evaluate the final accuracy reached by the system.

\section{A New CALIBration Algorithm}

The classic robot calibration is a procedure that permits to increase the final accuracy of a robot. In the literature it encompasses four distinct actions [8]:

1. Determination of the robot inverse geometric model,

2. Measure of several end-effector poses,
3. Mathematical identification of the difference between the geometric model found in 1 . and the real endeffector poses measured in 2 .,

4. Implementation of the model found in 3. in the robot controller,

In our experience we have noticed that this procedure is effective, but it is limited to cases where the wanted final accuracy is maximum $\sim 1 \mu \mathrm{m}$ [9]. This is not surprising, since this algorithm has been designed to compensate only the geometric errors of a robot.

When a sub-micrometric absolute accuracy is needed, it is necessary to consider other sources of inaccuracy that starts having an influence at this level. Those ones have to be identified, modeled and compensated constantly during the robot usage.

Consequently, we have added them in the classic calibration algorithm, establishing the following procedure for calibration of ultra-high-precision robots:

0. A design of the robot that takes into account the calibration problem and the pose measurement,

1. Identification of the sources of inaccuracy linked to the robot and the industrial process that it will perform,

2. Measure of several end-effector positions,

3. Identification of a function that describe the geometric model of the robot while is under the influence of the sources of inaccuracies identified in 1. ,

4. Implementation of the model found in 3 . in the robot controller,

5. Validation (evaluation of the robot absolute accuracy) and eventually return to 1 . (if the desired absolute accuracy has not yet been reached and consider other sources of inaccuracy), or return to 0 . (if all the possible sources of inaccuracy have already been considered).

While 0., 1. and 5. are original points, 2. and 4. remain equal to the ones from the classic procedure. Furthermore, step 3. is the fusion of the old steps 1 . and 3 .

Point 0 . does not really tackle the issue of calibration, but more the issue of robot design. It has been introduced here because when developing ultra-high-precision robots it is not a good attitude to split the robot conception by the calibration. Both problems have to be considered at the same time, in order to avoid cases where it is not possible to calibrate the robot for errors coming from the conception phase (e.g. cases where the end-effector is covered or unreachable from the measuring instruments, or cases where parasitic DOF not allow the use of certain measuring devices).

The remaining steps are detailed in the following paragraphs.

\section{SOURCES OF INACCURACY}

According to the literature, there are three kinds of sources of inaccuracy [10]: 
1. Geometric errors (caused by manufacturing tolerances, and assembling)

2. Non-geometric errors (caused by friction, backlash, wear, link compliances, joint encoder offsets and control errors)

3. Effects linked to the robot working conditions (caused by environmental factors, external forces acting on the robot and incertitude due to the reference position)

In the case of ultra-high-precision robots, geometric errors are compensated using the classic calibration technique. Non-geometric errors are avoided by designing the robot judiciously. Different effects acting on the robot during its use are described in the following points.

\section{A. Thermal Effects}

Thermal drift alone is one of the major causes of accuracy loss for ultra-high-precision robots [11]. Variations in the air temperature surrounding the robot will deform it and change its geometry.

One solution that has been adopted in the literature consisted in isolating the robot in a box where an air thermal stabilization is performed [12]. This solution is effective but not satisfactory in terms of time.

Thermal calibration (an active compensation of the thermal drift by modeling it) has already been used successfully to calibrate a linear axis [13] and the robot Agietron Micro-Nano [14].

Finally, we must consider that the robot itself can be a source of thermal variations: the designer of the robot has to keep in account that motors are responsible for heating the robot structure and deform it. This is why motors have to be placed in a spot where their thermal influence is not relevant.

\section{B. External Forces}

The forces generated by the manufacturing process can deform the robot as well [15]. Their influence in terms of loss of accuracy depends by their order of magnitude and by the stiffness of the robot. We have evaluated that for an ultra-high-precision robot, a force in the order of 1-2 $\mathrm{N}$ is responsible of an accuracy loss of $\sim 1 \mu \mathrm{m}$ [9].

Gravity or changes in the position of the center of mass can lead to an accuracy loss. About gravity, it is known that parallelograms (the basic structure used to perform 1 DOF translation in flexure hinges robots) deform in different ways dependently by their orientation toward the gravity vector [16]. An effect of this type will be seen for example in the case where the whole robot is rotated around a horizontal axis. On the other side, deformations due to change in the center of mass position can be observed in the case of pickand-place application, if the object displaced has a considerable mass.

\section{Reference Issues}

This issue has to be faced once the robot calibration is done. In fact, it is needed that the two robots are already able to compensate the thermal effects and the external forces effects.

This source of inaccuracy is related to the incertitude in positioning the tool-tip frame toward the frame that defines the end-effector pose (fig. 4 , the transformation from $\mathrm{C}_{\mathrm{A}}$ to $\mathrm{T})$. In fact, it is impossible to find a transformation matrix that describes such relation at ultra-high-precision level.

To allow the practical use of the robot, we suggest touching the part that will be manufactured by the robot with the tool-tip. The position where the contact happens defines a new frame that will be used to perform the manufacturing process.

In the case of multiple robots system, this problem is repeated for each robot. The complication here is due to the fact that a link between the frames of all the robots involved in the process has to be found as well (fig. 4, the transformation L). Since also in this case it is impossible to measure this relation, we suggest - again - to cause the contact of the two robots tool-tips and to define an origin in this point.

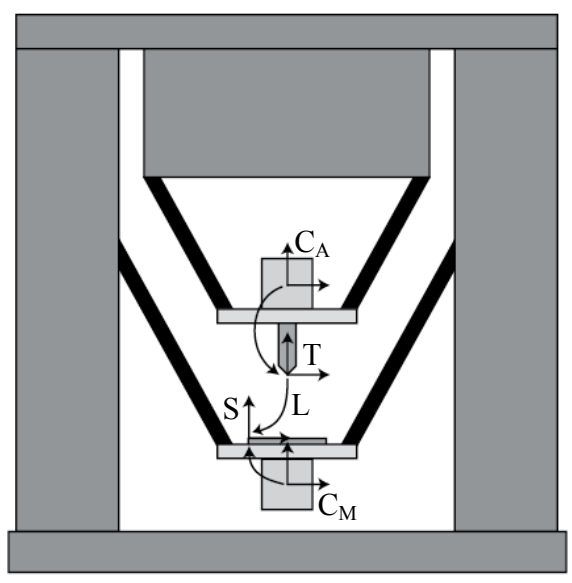

Fig. 4. The frames involved in the use of the system. $C_{A}$ and $C_{M}$ are the frames on the mirrored cubes used for the pose measurement. $\mathrm{T}$ and $\mathrm{S}$ are respectively the tool-tip and the substrate frames. L represents the link transformation between the $\mathrm{T}$ and $\mathrm{S}$ frame.

\section{CAlibration System}

The measuring devices are chosen dependently to the final accuracy needed. The resolution and the accuracy of the measuring devices must be lower than the resolution of the robot encoders and the desired final accuracy. As a good rule of thumb, a factor 10 between those values is at least required to perform good measurements.

Notice that the position/orientation measuring devices are used only to perform the measurement of the point II.3. On the other side, all the sensors that are used to measure the sources of inaccuracy (temperatures, forces, etc.) will be used also during the normal robot usage.

In the following sections we will describe the measuring system we have used to perform the calibration of the two robots system. 


\section{A. Measuring System for Position and Orientation}

A 6 DOF measuring system has been conceived to measure translations and rotations at very high-precision (fig. 5 and fig. 6). Even if each robot has only 3 DOF, we measure the 6 DOF so we are able to map the parasitic DOF of the robots.

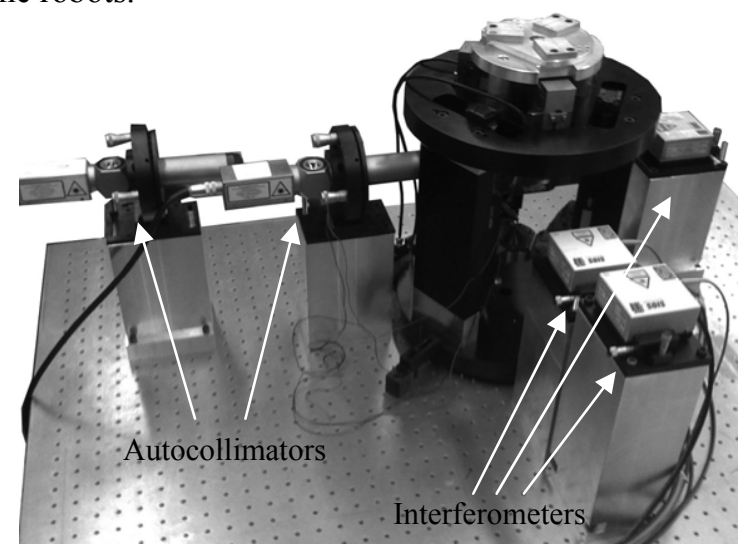

Fig. 5. The measuring system used to measure the pose of the Agietron Micro-Nano.

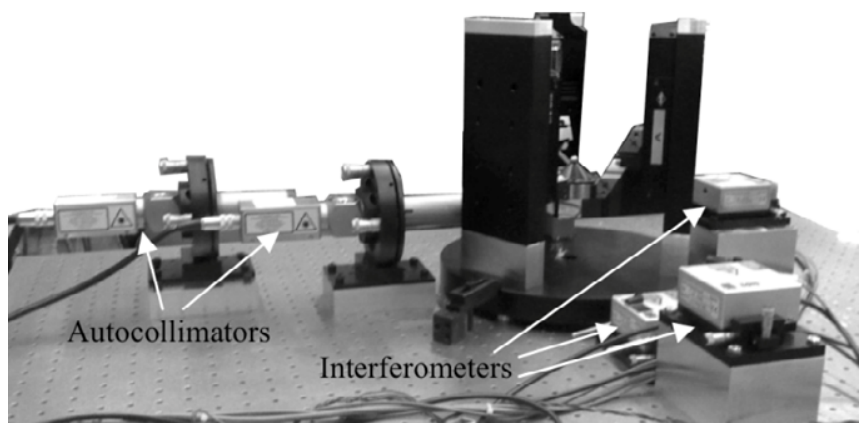

Fig. 6. The measuring system mounted to measure the pose of the MinAngle (in this picture the Agietron Micro-Nano is not mounted)

Translations are measured using 3 laser interferometers (SIOS SP-2000, resolution of $\sim 1.24 \mathrm{~nm}$, wavelength of $\sim 633$ $\mathrm{nm}$, stroke of $\sim 2 \mathrm{~m}$ ) mounted along the horizontal axes (a $45^{\circ}$ mirror is used to measure the vertical axis).

Rotations are measured using 2 autocollimators (Newport LDS-1000 Autocollimator, resolution of 0.02 arcsec, stroke of $\pm 400 \mathrm{arcsec}$ ), capable of measuring 4 DOF (the vertical rotation axis is measured by both devices, allowing to do measuring confirmation tests). The principal aim of the rotation measurement is to compensate the end-effector parasitic rotations of the Agietron Micro-Nano. Those rotations affect the interferometer reading, adding the so called cosine error [10]. Errors dues to parasitic rotations are corrected in real-time.

Each robot has been equipped with a 5 facets mirrored cube. Such cubes are used to reflect the laser beams of the measuring devices. Furthermore, each of them defines the origin of the robot and the orientation of its frame.

\section{B. Measurement of the Sources of Inaccuracy}

For studying how the thermal effects deform the robots, we have glued 7 thermal sensors (platinum resistance thermometers - pt1000) on the system. Meanwhile, we monitor the air temperature near the laser interferometer beam, using 3 thermal sensors pt100 (fig. 7). The 10 readings are acquired using a high-precision multi-channel A/D converter (Keithley 2700). Lastly, 3 pt1000 are mounted on each interferometer base. The reading of those sensors is used to stabilize the aluminum interferometer bases, using a Peltier cell controlled with a PID logic. This is done to assure that the interferometers are not subject to thermal drift [13].

For the case of nano-indentation, we are not going to measure and compensate the forces generated by the process. This is because we have evaluated that the forces generate by the process are around $10^{-3} \mathrm{~N}$. As we said before, since 1-2 $\mathrm{N}$ causes $\sim 1 \mu \mathrm{m}$ drift, we decide not to complicate the model (and the measuring system, adding a force sensor) because we would have gained anything relevant in absolute accuracy.

Force compensation in the case $\mu$-EDM process has to be done if sub-micrometric accuracy is demanded. Such subject has exhaustively been covered in the article [17].

\section{Measurement Procedure}

The measurements are done separately for each robot. While measuring is in course, the sources of inaccuracy must be acting on the robot. In this way it is possible to measure their effect on the robot geometry.

It is recommended to measure the pose of the end-effector in all the workspace, doing a regular trajectory. Furthermore, it is necessary to collect some separate measurement that will be used to validate the calibrated model once found. Those measures have imperatively to not be used to calibrate the robot.

For mapping the thermal drift, it is important to vary the temperatures acting on the robot in the same range of temperatures that the robot will encounter during is normal use. For mapping external forces, it is important to use a force captor to evaluate the force vector acting on it. If it is possible, also the reading of the force used to control robot actuators can be use to model the force behavior.

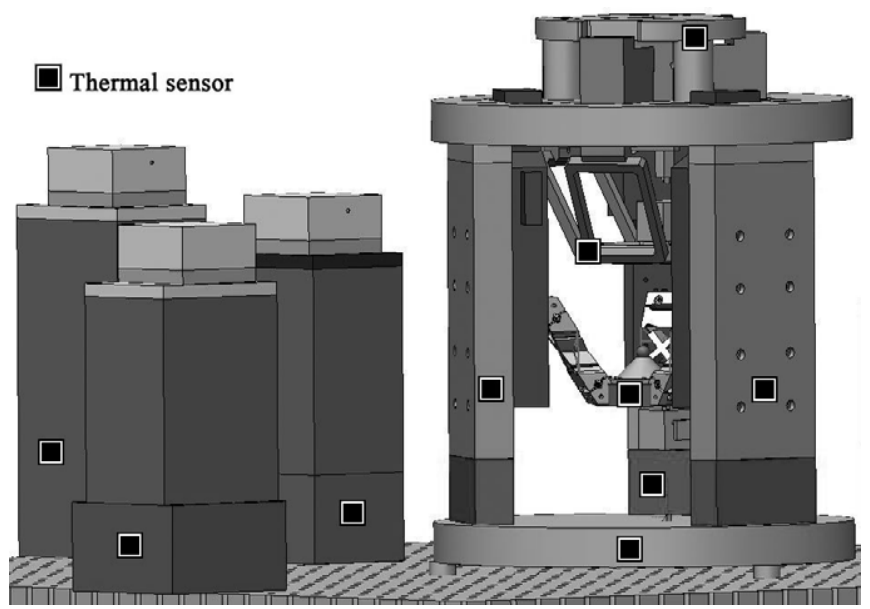

Fig. 7. Positions of the thermal sensors on the system. 


\section{Indentation System}

After the calibration we will use nano-indentation to evaluate the absolute accuracy of the system. The components used to do it are mounted before the measurement: mounting them after will change the center of gravity of the two end-effectors, invalidating the measures.

We have equipped the Agietron Micro-Nano with a Vickers diamond and the MinAngle with a substrate in Invar $^{\circledR}$ (fig. 8), to avoid thermal drift of the substrate (Invar has a very low thermal expansion coefficient). After the calibration, several marks will be indented on the substrate in well known positions. The marks positions will finally be measured on a SEM microscope, in order to evaluate the absolute accuracy of the system.

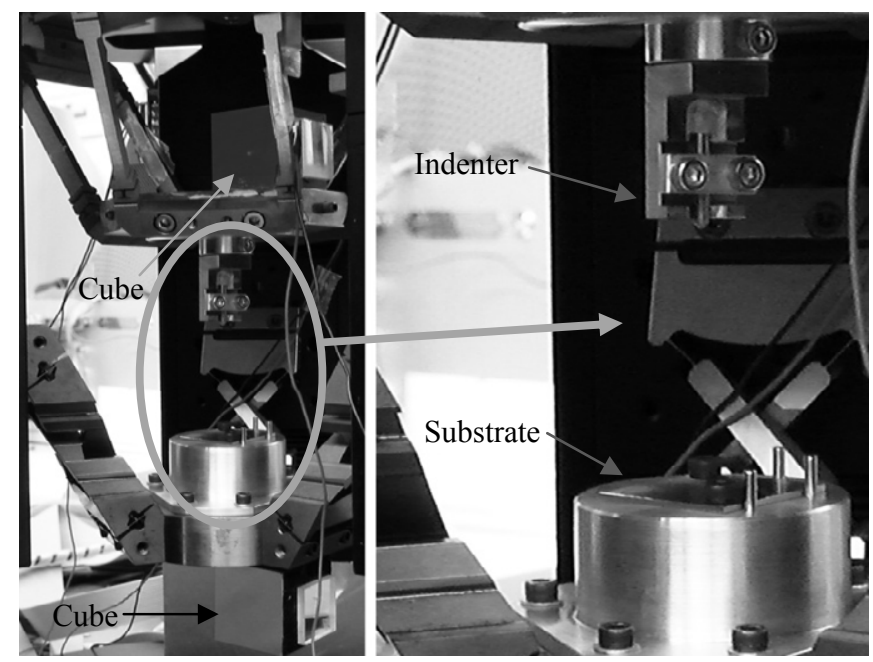

Fig. 8. The system to perform nano-indentation

\section{DATA PROCESSING}

Using the data collected before, we will build a model of the system that will describe the robots geometry and will keep in account the sources of inaccuracy that we have considered.

Between all the possible algorithms to perform data-fitting tasks, we have chosen the Stepwise Regression [18]. It has been chosen for two reasons: firstly it automatically deletes useless parameters, keeping the robot model computationally fast. Secondly, the algorithm converges and gives a solution in some seconds. On the contrary, algorithms tested in previous works (neural networks, gradient descent based parameters research, genetic algorithms and splines optimization, all discussed in [10]) take some hours to give a solution.

The algorithm has the capability of adding or removing terms from a multi-linear model. This is done by comparing the statistical significance of the terms in a regression. It starts with an initial model that is compared with larger or smaller models. At each step, a coefficient is added to the model, thus, it is compared the final error with or without this last coefficient. If there is an improvement in the prediction, the coefficient is kept. Otherwise the coefficient is discarded. For the coefficients that are already in the model it happens the same: if the influence of a coefficient is under a certain threshold, the coefficient is rejected.

Depending on the terms included in the initial model and the order in which terms are moved in and out, the method may build different solutions from the same set of terms. The method terminates when any single step improves the model prediction capability. There is no guarantee that a different initial model or a different sequence of steps will not lead to a better fit. In this sense, stepwise models are locally optimal, but may not be globally optimal.

\section{AdDing The CAlibrated Model To The Robot CONTROLLER}

The model found in the last phase has finally to be implemented in the controller. This model can be really complex, depending by the number of sources of inaccuracy modeled. From a computational point of view, robot inverse geometric models are calculated in a real-time process. To fast its calculation, it is possible to detach the thermal coefficient by the others. Thermal variations are very slow, so they can be calculated in a non-real-time process each 510 seconds. On the other side, variations dues to external forces have imperatively to be compensated in real-time.

\section{VALIDATION AND RESULTS}

Here we present the results that we had for the calibration of the two robots, thus the indentation results.

\section{A. Offline Calibration Results}

The offline absolute accuracy for each robot is calculated by predicting a data set of points that has not been used to perform the calibration: the validation set. In predicting it, the Agietron Micro-Nano obtained an accuracy of $\pm 70 \mathrm{~nm}$ in motor coordinates in the $90 \%$ of the set, equivalent to $1.645 \sigma$. We found similar values in measuring the error in end-effector coordinates. The model used for this robot has 78 parameters (45 geometric and 33 thermal). The parasitic rotations have been also measured and modeled (on the three axes, the parasitic rotations never exceed \pm 50 ' ').

With the system in our possession it has been possible to calibrate the MinAngle with ultra-high-precision only while performing pure translations along the vertical axis. In this case, we obtained a measured absolute accuracy of $\pm 1 \mu \mathrm{m}$. As the interferometer cannot handle rotation wider than 200 ', we used a touch probe to measure the robot position while rotating. As the touch probe introduces dry friction in the system, we had measures with a hysteresis that allow us to reach an absolute accuracy of $\pm 5 \mu \mathrm{m}$ in motor coordinates (corresponding to \pm 15 ', in end-effector coordinates).

The MinAngle model is composed by 63 parameters (of which only 3 are thermal and used to model the drift measured with the interferometer along the vertical axis). Furthermore, the parasitic translation of the MinAngle have been measured and modeled (while moving along the vertical axis, the robot perform a total parasitic translation of $12 \mu \mathrm{m}$ along the horizontal axes). 


\section{B. Reference compensation}

As seen in III.C, to use the robots at the same time it is necessary to relate their frames. In this case, this is done by acting on two aspects.

Firstly, the model of the MinAngle parasitic translations is inserted in the controller of the Agietron Micro-Nano. In this way the Agietron Micro-Nano is able to compensate the translations performed by the MinAngle while it is moving along the vertical axis.

The second operation consisted in aligning the axes of the two frames. To do so, we used the indenter to touch the substrate in three points (fig. 9). In this way it has been possible to define the substrate plane and calculate the difference in orientation between it and the frame of the Agietron Micro-Nano. Thus, the MinAngle end-effector has been rotated in order to correct such difference. Finally, we define the first indent as the zero of the nano-indentation process. In this way, all the errors dues to misalignment are avoided.

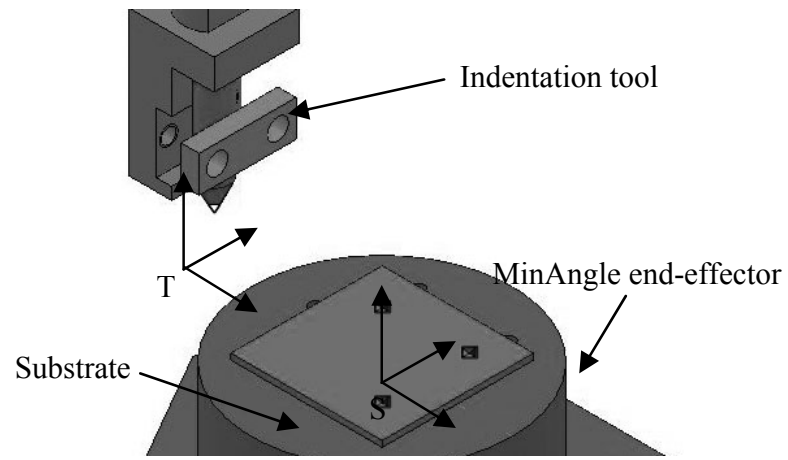

Fig. 9. The frames used to perform nano-indentation: $\mathrm{T}$ is the tool-tip frame of the Agietron Micro-Nano and S is the frame of the substrate (coincident with the first indent) mounted on the MinAngle end-effector.

\section{Indentation Results}

Ten marks have been indented in the center of the substrate. One in the center will be considered as the zero for the measures in the microscope (fig. 10). Three marks have been indented at regular intervals of $0.05,0.1$ and $0.2 \mathrm{~mm}$, along three lines centered on the zero, rotated between them of $120^{\circ}$.

A FEI XLF-30 EBSP SEM has been used to measure the absolute position and depth of the indents center. The microscope has an electronic optics resolution of 1.1 nm @ $15 \mathrm{kV}$. The horizontal navigation axes have a resolution of $100 \mathrm{~nm}$. As it is possible to see in fig. 10, we are able to indent marks with a clean shape, where the mark center is easily measurable.

We will then evaluate the absolute position of the other marks in respect to the zero mark, by using a different electronic microscope with horizontal navigation axes with a resolution of $1 \mathrm{~nm}$.

\section{CONCLUSION}

In this article we have introduced a reliable calibration algorithm suitable for ultra-high-precision robots operating in unsteady conditions. Through the calibration of a two robot system, we have demonstrated that it is effective.

Thus, we have introduced the nano-indentation as a new manner to evaluate the final absolute accuracy of the system.

In future work, we will develop a calibration technique that will directly keep in account indentation to collect the data for calibration.

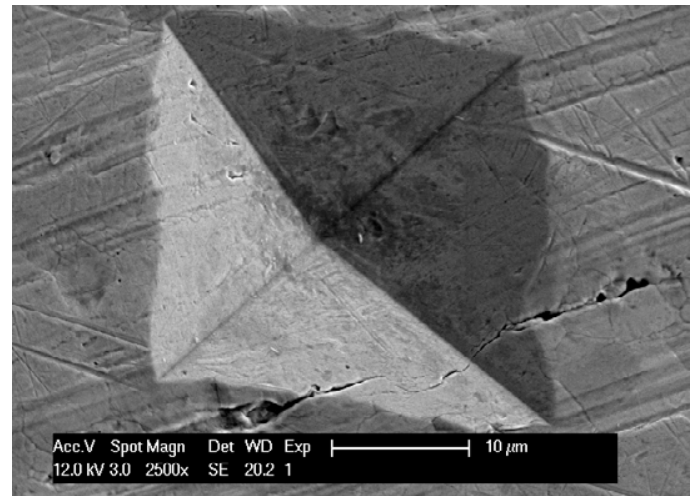

Fig. 10. A SEM picture of a mark done with the nano-indentation system.

\section{REFERENCES}

[1] I. Verettas et al. PocketFactory: a modular and miniature assembly chain including a clean environment. Besançon, IWMF, 2006.

[2] L. Everett et al. A Sensor Used for Measurements in the Calibration of Production Robots. Atlanta, IEEE, 1993.

[3] R. Clavel. Conception d'un robot paralléle rapide à 4 degrés de liberté. PhD Thesis n. 925, Lausanne, EPFL, 1991.

[4] C. Joseph. Contribution à l'accroissement des performances du processus de $\mu E D M$ par l'utilisation d'un robot à dynamique élevée et de haute precision. PhD Thesis n. 3281, Lausanne, EPFL, 2005

[5] P. Pham et al. Orion MinAngle: A flexure-based, double-tilting parallel kinematics for ultra-high precision applications requiring high angles of rotation. Tokyo, ISR Proceedings, 2005.

[6] A. Descoeudres. Characterization of electrical discharge machining plasmas. PhD Thesis n. 3542, Lausanne, EPFL, 2006.

[7] S.P. Baker. Nanoindentation Techniques. Encyclopedia of Materials: Science and Technology, pp 5908 - 5916, Elsevier, 2001.

[8] H. Zhuang et Al. Camera-Aided Robot Calibration. Boca Raton, CRC Press, 1996.

[9] E. Lubrano et Al. Compensation of thermal effects and cutting-forces acting on ultra high-precision robots. Bremen, Actuator 2010, 2010.

[10] N. Fazenda. Calibration of high-precision flexure parallel robots. PhD Thesis n. 3712, Lausanne, EPFL, 2007.

[11] R. Ramesh et al. Error compensation in machine tools - a review. Part II: thermal errors. Singapore, Pergamon, 2000.

[12] N. Fazenda et al. Calibration of the 6 DOF high-precision flexure parallel robot "Sigma 6". Chemnitz, Fraunhofer, 2006.

[13] E. Lubrano et al. Thermal behavior of an ultra high-precision linear axis operating in industrial environment. Bergamo, 2008.

[14] E. Lubrano et al. Thermal Calibration of a 3 DOF Ultra HighPrecision Robot Operating in Industrial Environment. Anchorage, IEEE, 2010.

[15] R. Ramesh et al. Error compensation in machine tools - a review. Part I: geometric, cutting-force induced and fixture-dependent errors. Singapore, Pergamon, 2000.

[16] T. Niaritsiry. Optimisation de la conception du robot parallèle delta cube de très haute précision. PhD Thesis n. 3567, Lausanne, EPFL, 2006.

[17] E. Lubrano et al. Modeling and compensation of cutting-forces generated during the EDM process for ultra high-precision robots. Munich, ISR proceeding, 2010.

[18] The MathWorks ${ }^{\mathrm{TM}}$ Statistics Toolbox ${ }^{\mathrm{TM}}$ 6, User guide. The MathWorks ${ }^{\mathrm{TM}}, 2008$. 